\title{
Numerical Modeling of Radiation Focusing by Dielectric Microcylinders with Several Layers
}

\author{
Alexandra A. Savelyeva ${ }^{1,2^{*}}$ and Elena S. Kozlova ${ }^{1,2}$ \\ ${ }^{1}$ IPSIRAS - branch of the FSRC “Crystallography and Photonics" RAS, 151 Molodogvardejskaya str., Samara 443001, \\ Russia \\ ${ }^{2}$ Samara National Research University, 34A Moskovskoe Shosse, Samara 443086, Russia \\ *e-mail: lexis2450@gmail.com
}

\begin{abstract}
In this paper, the light focusing by dielectric microcylinders with several layers was numerically studied by using the finite element method implemented in COMSOL Multiphysics. Different materials for the top layer were proposed during the research (refractive indices are 1.59, 1.8, 1.9) while the core was proposed from fused silica glass (refractive index is 1.45). It was shown that the dielectric shell allows to increases the maximum intensity of the formed photonic nanojet. Choosing the design of a two-layered microcylinder it is possible to form a compact focal spot (TE-polarization) or increase the length of the produced photonic nanojet. Obtained results were verified by another software package FullWAVE which implements a finite difference time domain method. The simulation results are in good agreement. (C) 2021 Journal of Biomedical Photonics \& Engineering.
\end{abstract}

Keywords: two-layered dielectric microcylinder; photonic nanojet; sharp focusing; FDTD-method; FEM-method.

Paper \#3434 received 25 May 2021; revised manuscript received 21 Sep 2021; accepted for publication 22 Sep 2021; published online 30 Sep 2021. doi: 10.18287/JBPE21.07.030304.

\section{Introduction}

Compression of light into a narrow beam is one of the priority tasks in nanophotonics [1-3]. Ultra-narrow light spots or nanojets are widely used in modern optical instrumentation. The problems of nanojets formation and optimization of their parameters are being actively studied by the world scientific community due to their importance in different areas [3-5] and mostly in biomedicine [6-8]. They are used for micromanipulation [9, 10], optical tweezers [11-13], for fluorescence enhancement in Raman spectroscopy [14], in histological studies [15], in vivo application [16].

A nanojet is an ultra-narrow light spot spreading over several wavelengths [17-20]. The main parameters which are primarily of interest to researchers are the maximum intensity, the distance from the optical element to the peak of the intensity, the full width (FWHM) and depth (DOF) at half maximum of intensity. In their research, scientists try to optimize the nanojet in such a way as to obtain the most compact light spot with maximum intensity in focus and maximum length. There are many articles on the generation and optimization of nanojets using different optical elements [17-26]. In study [18] authors report about narrow photonic nanojet which is formed by an engineered two-layer microcylinder of high refractive-index materials. Finite element analysis showed that under $632.8 \mathrm{~nm}$ illumination, the full width at half maximum of the beam waist can reach $87 \mathrm{~nm}$. In Ref. [21], using numerical simulations it was shown that when focusing light with a wavelength of $\lambda=550 \mathrm{~nm}$ by a micro-flat-ended cylinder with a refractive index $n=4$, the full width at half maximum can reach $70 \mathrm{~nm}$. Using the COMSOL Multiphysics software package it was shown in Ref. [23], that a triangular prism made of quartz glass and illuminated by laser light with wavelength of $4 \mu \mathrm{m}$ forms a focal spot the maximum intensity of which exceeds the intensity of the incident radiation for 5 times. The FWHM of obtained nanojet was $0.38 \lambda$. In Ref. [26], for the first time, authors numerically demonstrate that the size of the nanojet formed in the localized region of the dielectric mesoscale sphere can be significantly reduced by introducing a nanohole at its shadow surface.

The improvements are often made to the design of the focusing optical element for optimization of the nanojet parameters. For example, metal cores $[27,28]$ and shells [29, 30], multilayer dielectric structures [31, 32] can be used. Two types of spheres: an ordinary dielectric 
sphere and a sphere with a metal core are considered in Ref. [27]. The best result in light focusing was achieved using a two-layered sphere. In Ref. [28], the authors proposed a method for light focusing by multilayer cylinders with metal shells made of gold, silver, and copper. It is shown that metal coatings affect the location of the nanojet. For a microcylinder made of gold and silver, a focal spot with FWHM $=293 \mathrm{~nm}$ was obtained. In Ref. [30], we proposed an unusual design of a polyester cylinder with silver film and a gap on the shadow side. A series of numerical simulations were carried out in which the hole diameter was varied. It was shown that a microcylinder with a radius of $2.1749 \lambda$ and with a silver film of $90 \mathrm{~nm}$ and a hole of $100 \mathrm{~nm}$ formed a light field with a maximum intensity of 10.61 a.u. which is 1.4 times higher than the intensity of light field formed by the ordinary polyester cylinder. In Ref. [31] the authors consider five-layer cylinders as a focusing elements. The selection of radius and refractive indices was carried out using a genetic algorithm. The research showed that the parameters of the generated nanojets can be controlled by changing the focusing elements design. For example, a five-layer microcylinder designed in this study managed to generate an ultra-long nanojet with length of $107.5 \lambda(\lambda=632.8 \mathrm{~nm})$.

In this paper, we investigate the focusing process of TE- and TM-polarized electromagnetic light with a wavelength $\lambda=633 \mathrm{~nm}$ by two-layered circular dielectric microcylinders. In the first part of the study, the influence of dielectric shell on photonic nanojet formation by using numerical experiments in COMSOL Multiphysics 5.1 which implements the finite element method (FEM) was evaluated. The two-layered structure of microcylinders is optimized. In the second part, the obtained results were verified by another software package FullWAVE 2020.03 which implements the finite difference time domain method (FDTD method). It showed good agreement with COMSOL Multiphysics.

\section{Materials and methods}

In our research, we discuss the process of linearly polarized laser light focusing by two-layered dielectric microcylinders. The wavelength $\lambda$ was chosen equal to $633 \mathrm{~nm}$. The total cylinder diameter is fixed at $2 \mu \mathrm{m}$, and the core diameter $\left(D_{\text {core }}\right)$ is varied from $1.2 \mu \mathrm{m}$ to $1.9 \mu \mathrm{m}$. The refractive index of the cylinder core is $n_{1}$, and the refractive index of the cladding is $\mathrm{n}_{2}$. The numerical simulation scheme is shown in Figure 1, where $D_{\text {core }}$ is the core diameter and $D_{\text {sh }}$ is the shell diameter of cylinder.

This paper demonstrates the results of the numerical simulations obtained using the software package COMSOL Multiphysics which implements FEM [33, 34] for solving next wave equation:

$$
\nabla \times \mu_{r}^{-1}(\nabla \times \boldsymbol{E})-k_{0}^{2}\left(\epsilon_{r}-\frac{j \sigma}{\omega \epsilon_{0}}\right) \boldsymbol{E}=0
$$

where $\mu_{r}$ is the relative permeability, $\boldsymbol{E}$ is the vector of electric field intensity, $k_{0}$ is the free-space wavenumber, $\varepsilon r$ is the relative permittivity, $j$ is the imaginary unit, $\sigma$ the electrical conductivity, $\omega$ is the angular frequency of incident light, $\varepsilon_{0}$ is the permittivity of vacuum. This method is widely used in various studies of light focusing [35-37].

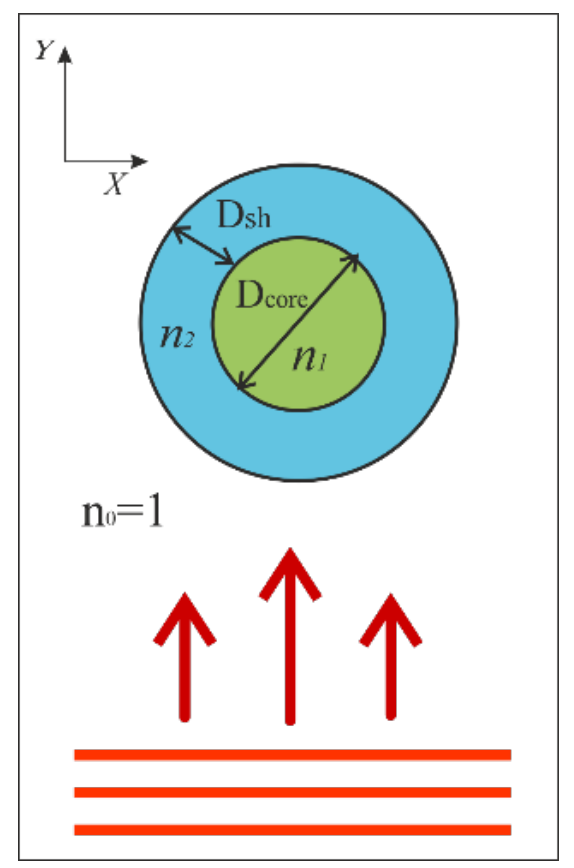

Fig.1 - Scheme of numerical simulation.

A triangular mesh with the following parameters were used in modeling: a step of $\lambda / 60$ for areas where the refractive index changes and $\lambda / 40$ for regions with constant refractive index. These steps provide numerical convergence (standard deviation is $0.001 \%$ ).

After the main simulation results were obtained by using the COMSOL Multiphysics, a series of additional simulations was made for the cylinders with the best designs. For additional simulations FDTD-method implemented in the FullWAVE software package from RSoft Design Group was applied. This algorithm used the Maxwell's system of equations [38]:

$$
\left\{\begin{array}{l}
\frac{\partial \boldsymbol{D}}{\partial t}=\operatorname{rot} \boldsymbol{H} ; \\
-\frac{\partial \boldsymbol{B}}{\partial t}=\operatorname{rot} \boldsymbol{E}
\end{array}\right.
$$

where $\boldsymbol{H}$ is vector of the magnetic field, $\boldsymbol{D}$ and $\boldsymbol{B}$ are vectors of the electric and magnetic induction.

FDTD method was firstly proposed by Kane S. Yee in Ref. [39]. Currently, this method is widely used by scientists in various studies, for example in Refs. [40 - 44].

The following parameters of a rectangular grid in space were used here: a $20 \mathrm{~nm}$ step along transverse and longitudinal coordinates. The temporal step $c \Delta t$ was chosen equal to $10 \mathrm{~nm}$ according to Courant condition. These steps ensure the convergence of the numerical method (the standard deviation is $0.001 \%$ ). While analyzing simulation results the averaging of the intensity distribution for 10 periods was made. 


\section{Results and Discussion}

\subsection{Numerical simulation using FEM-method}

In this section, the main study obtained by using numerical simulations in the software package COMSOL Multiphysics is presented. Firstly, TE-polarization is investigated. We considered microcylinders with core made of fused silica glass $\left(\mathrm{n}_{1}=1.45\right.$ [45]) and shell made of $\operatorname{SiN}\left(\mathrm{n}_{2}=1.9\right.$ [46]). During the simulation results analysis, we evaluate the following parameters of the nanojet: the maximum intensity $I_{\max }$, the focal length $F$, FWHM, and DOF, which are presented in Table 1.

It can be seen from Table 1 that the best design of a two-layered cylinder is the case when $\mathrm{D}_{\text {core }}=1.7 \mu \mathrm{m}$. The results for this microclinder are marked as bold. Despite the fact that the maximum intensity of the nanojet is observed for a simple dielectric cylinder with a refractive index of $\mathrm{n}_{1}=1.45$, a two-layer cylinder makes it possible to increase DOF by about 2 times. This fact can simplify the use of such nanojets in applications.

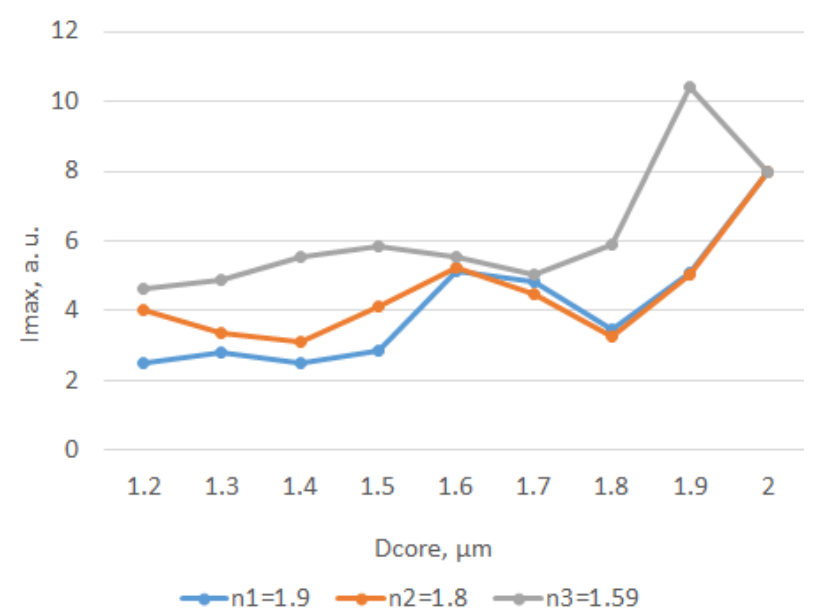

(a)

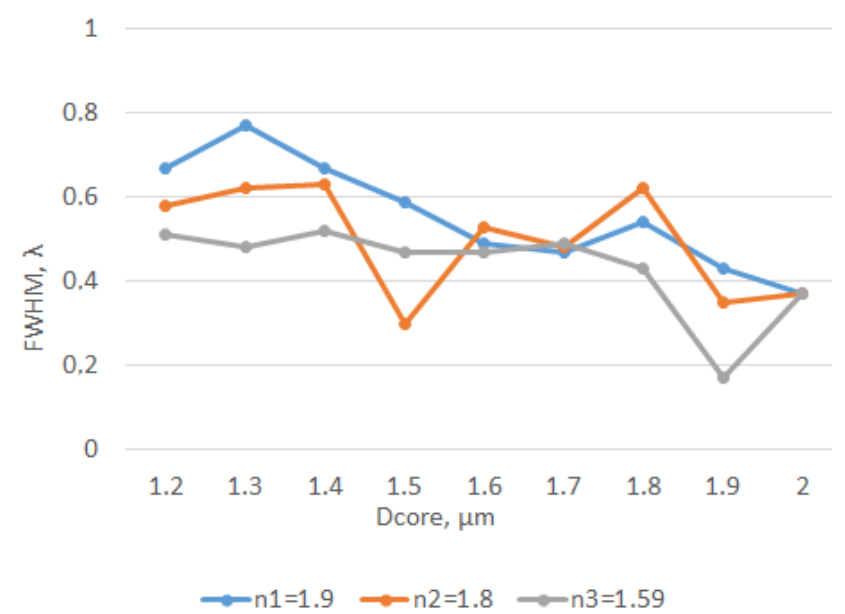

(c)
Table 1 Parameters of the focal spot formed by the twolayered microcylinders with $\mathrm{n}_{1}=1.45$ and $\mathrm{n}_{2}=1.9$.

\begin{tabular}{ccccc}
$\mathbf{D}_{\text {core, }} \boldsymbol{\mu \mathbf { m }}$ & $\mathbf{I}_{\mathbf{m a x}}, \mathbf{a . u}$. & $\mathbf{D O F}, \boldsymbol{\lambda}$ & $\mathbf{F W H M}, \boldsymbol{\lambda}$ & $\mathbf{F}, \boldsymbol{\mu m}$ \\
\hline 2.0 & 7.97 & 0.78 & 0.37 & 0.02 \\
\hline 1.9 & 5.10 & 0.91 & 0.43 & 0.13 \\
\hline 1.8 & 3.44 & 1.32 & 0.54 & 0.21 \\
\hline 1.7 & 4.81 & 1.66 & 0.47 & 0.07 \\
\hline $\mathbf{1 . 6}$ & $\mathbf{5 . 1 3}$ & $\mathbf{1 . 7 9}$ & $\mathbf{0 . 4 9}$ & $\mathbf{0 . 2 5}$ \\
\hline 1.5 & 2.87 & 2.35 & 0.59 & 0.62 \\
\hline 1.4 & 2.51 & 2.05 & 0.67 & 0.52 \\
\hline 1.3 & 2.83 & 2.23 & 0.77 & 0.60 \\
\hline 1.2 & 2.51 & 2.05 & 0.67 & 0.82
\end{tabular}

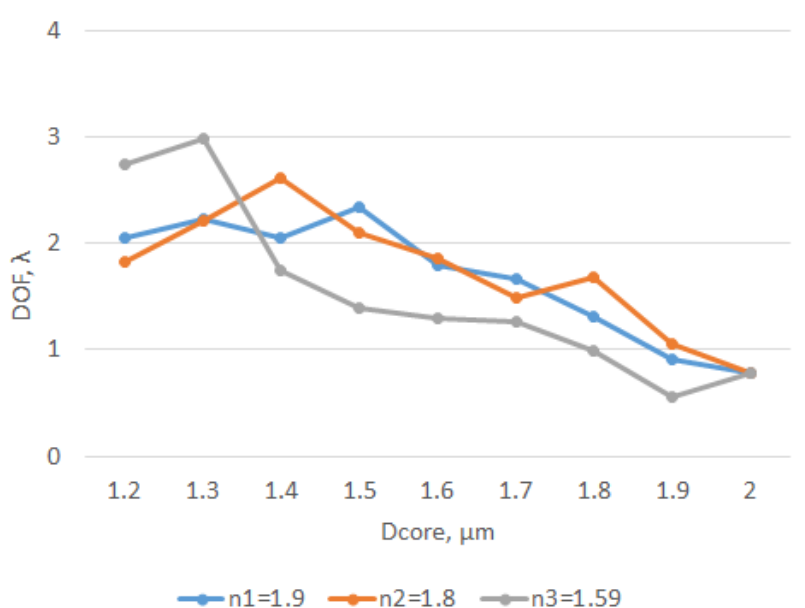

(b)

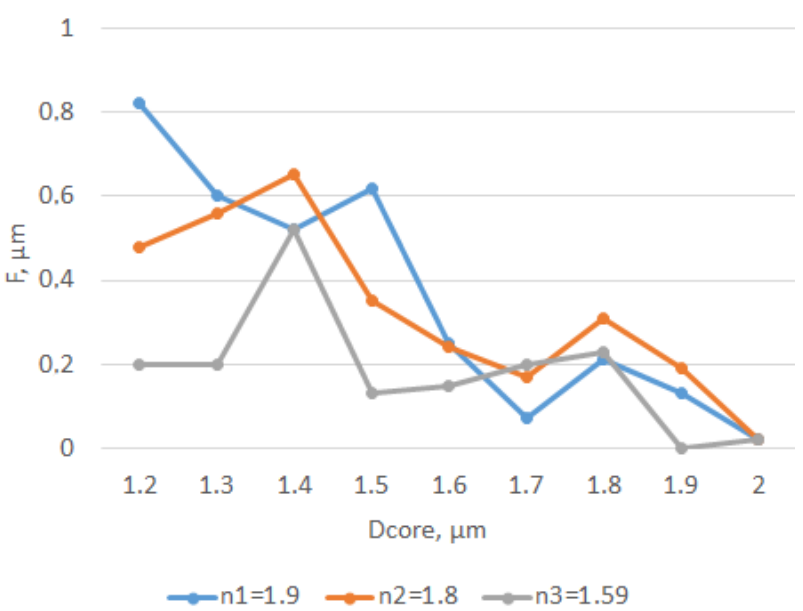

(d)

Fig. 2 Dependence of the $I_{\max }$ (a), DOF (b), FWHM (c), F (d) on the core diameter for TE-wave. 


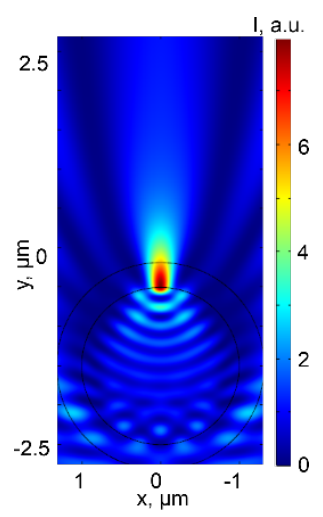

(a)

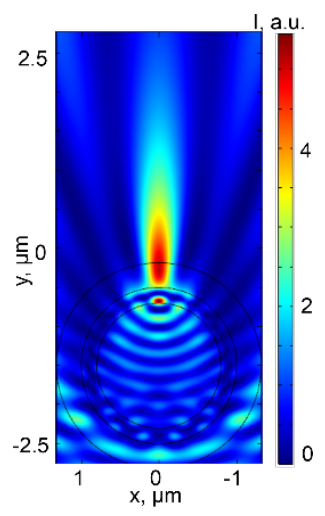

(b)

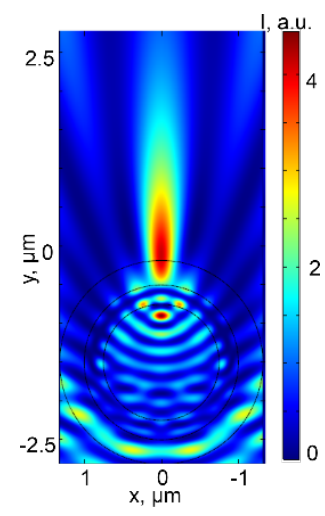

(c)

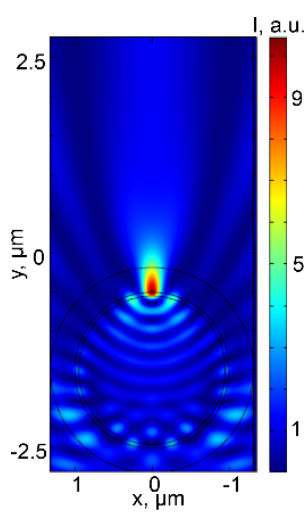

(d)

Fig. 3 The intensity distribution for ordinary microcylinder (a) and intensity distribution for two-layered microcylinders with $\mathrm{n}_{2}=1.9$ and $\mathrm{D}_{\text {core }}=1.6(\mathrm{~b}) ; \mathrm{n}_{2}=1.8$ and $\mathrm{D}_{\text {core }}=1.5$ (c); $\mathrm{n}_{2}=1.59$ and $\mathrm{D}_{\text {core }}=1.9$ (d) illuminated by TE-wave.

Next, we propose the shell from titanium oxideorganic polymer $\left(\mathrm{n}_{2}=1.8\right.$ [47]). The obtained results are presented in Table 2.

Table 2 Parameters of the focal spot formed by the twolayered microcylinders with $\mathrm{n}_{1}=1.45$ and $\mathrm{n}_{2}=1.8$.

\begin{tabular}{ccccc}
$\mathbf{D}_{\text {core, }} \boldsymbol{\mu m}$ & $\mathbf{I}_{\text {max }}, \mathbf{a . u}$. & $\mathbf{D O F}, \boldsymbol{\lambda}$ & $\mathbf{F W H M}, \boldsymbol{\lambda}$ & $\mathbf{F}, \boldsymbol{\mu m}$ \\
\hline 2.0 & 7.97 & 0.78 & 0.37 & 0.02 \\
\hline 1.9 & 5.06 & 1.06 & 0.35 & 0.19 \\
\hline 1.8 & 3.26 & 1.69 & 0.62 & 0.31 \\
\hline 1.7 & 4.47 & 1.49 & 0.48 & 0.17 \\
\hline 1.6 & 5.24 & 1.86 & 0.53 & 0.24 \\
\hline $\mathbf{1 . 5}$ & $\mathbf{4 . 1 3}$ & $\mathbf{2 . 1 0}$ & $\mathbf{0 . 3 0}$ & $\mathbf{0 . 3 5}$ \\
\hline 1.4 & 3.10 & 2.61 & 0.63 & 0.65 \\
\hline 1.3 & 3.34 & 2.22 & 0.62 & 0.56 \\
\hline 1.2 & 4.01 & 1.82 & 0.58 & 0.48
\end{tabular}

From Table 2 we can see that the best design is represented by a two-layer cylinder with $\mathrm{D}_{\text {core }}=1.5 \mu \mathrm{m}$. It can be noticed that the two-layered cylinder does not give such a high intensity in the focal spot as ordinary cylinder, but nevertheless, the depth of the formed focus is much higher than that is for the other presented models.

At last, we simulate similar cylinders, but for the shell made of polystyrene $\left(\mathrm{n}_{2}=1.59\right.$ [45]). The results are presented in Table 3.

From Table 3 we can see that a two-layered cylinder with a core diameter $\mathrm{D}_{\text {core }}=1.9 \mu \mathrm{m}$ allows the formation of the tight focus with the best parameters: high intensity and smallest FWHM and DOF.

To simplify the analysis of the obtained results we presented the dependence of focal spot characteristics in Fig. 2.

It can be seen from Fig. 2 that all shells lead to increasing of the focal length, FWHM and DOF. However, in each case of different materials for shell
DOF (Fig. 2(b)) grows faster than FWHM (Fig. 2(c)). The maximum intensity is decreasing while using thick coatings (Fig. 2(a)).

Table 3 Parameters of the focal spot formed by the twolayered microcylinders with $\mathrm{n}_{1}=1.45$ and $\mathrm{n}_{2}=1.59$.

\begin{tabular}{ccccc}
$\mathbf{D}_{\text {core, }} \boldsymbol{\mu \mathbf { m }}$ & $\mathbf{I}_{\mathbf{m a x}}, \mathbf{a . u}$. & $\mathbf{D O F}, \boldsymbol{\lambda}$ & $\mathbf{F W H M ,} \boldsymbol{\lambda}$ & $\mathbf{F}, \boldsymbol{\mu m}$ \\
\hline 2.0 & 7.97 & 0.78 & 0.37 & 0.02 \\
\hline $\mathbf{1 . 9}$ & $\mathbf{1 0 . 4 3}$ & $\mathbf{0 . 5 6}$ & $\mathbf{0 . 1 7}$ & $\mathbf{0 . 0 0}$ \\
\hline 1.8 & 5.91 & 0.99 & 0.43 & 0.23 \\
\hline 1.7 & 5.06 & 1.27 & 0.49 & 0.20 \\
\hline 1.6 & 5.56 & 1.30 & 0.47 & 0.15 \\
\hline 1.5 & 5.83 & 1.39 & 0.47 & 0.13 \\
\hline 1.4 & 5.52 & 1.75 & 0.52 & 0.52 \\
\hline 1.3 & 4.88 & 2.98 & 0.48 & 0.20 \\
\hline 1.2 & 4.62 & 2.74 & 0.51 & 0.20
\end{tabular}

Distributions of intensity obtained while focusing of TE-polarized light by ordinary cylinder with $\mathrm{n}_{1}=1.45$ and by two-layered cylinders with optimal ratio of core and shell are presented in Fig. 3.

It can be seen from the Fig. 3 that the nanojet for a two-layer cylinder has elongated shape in case of $\mathrm{n}_{2}$ equal to 1.9 (Fig. 3(b)) and 1.8 (Fig. 3(c)). Moreover, it is formed at some distance from cylinder boundary. From the analysis of the Fig. 3(d) we can conclude that the dielectric shell with a refractive index which is a bit higher than the core refractive index allows to reduce the geometric dimensions of the focal spot and increase the intensity in focus.

Then we present series of numerical experiments for the same designs of microcylinders, but for TM-polarized plane wave as incident light. We briefly described the obtained results which are shown in Tables 4-6 and in Fig. 4. 


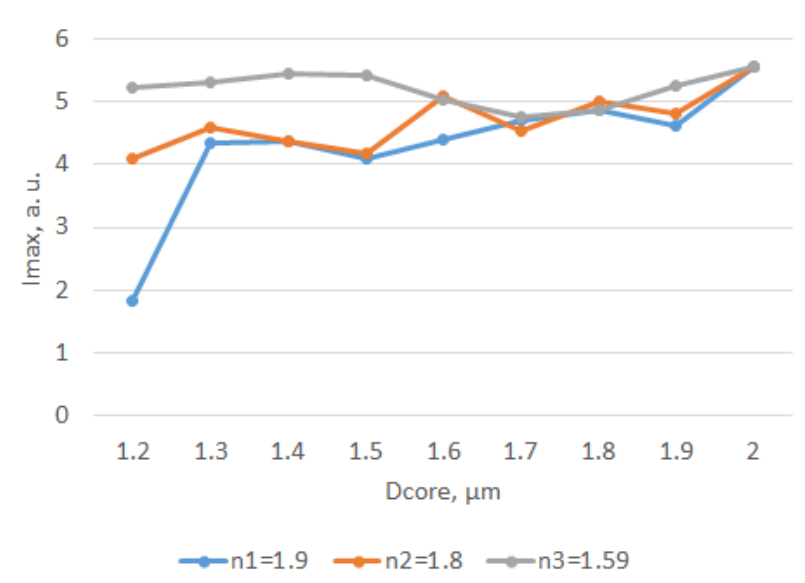

(a)

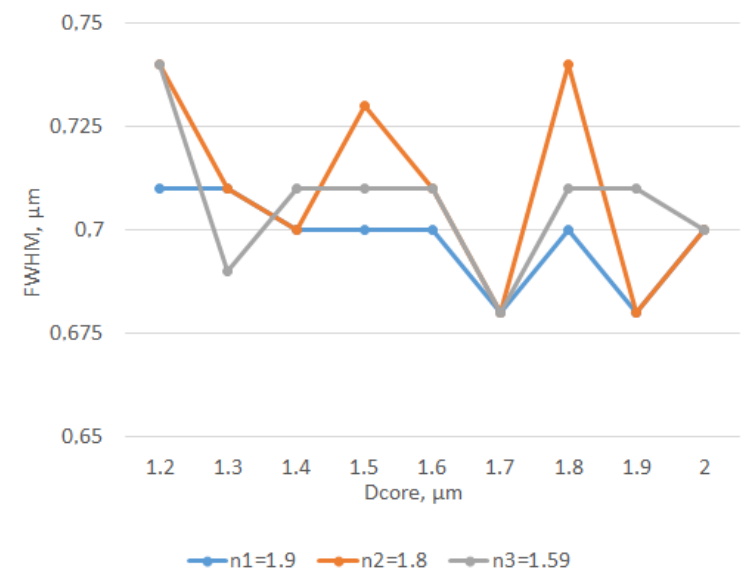

(c)

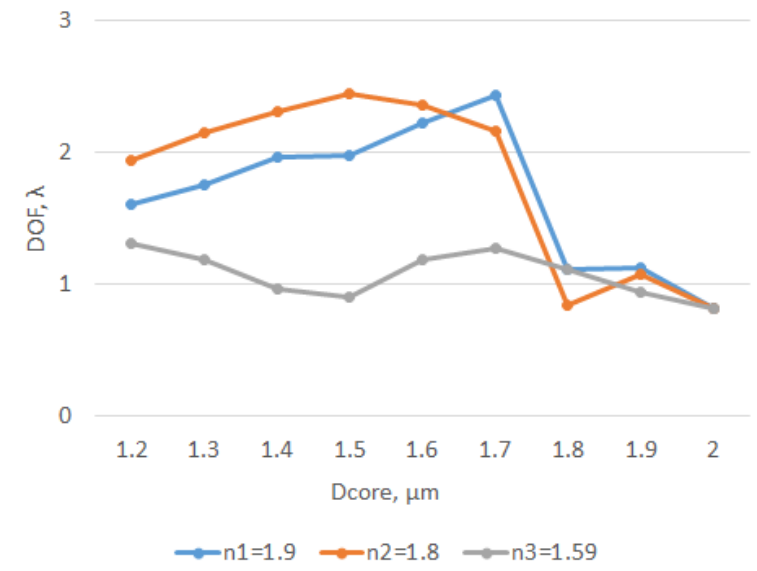

(b)

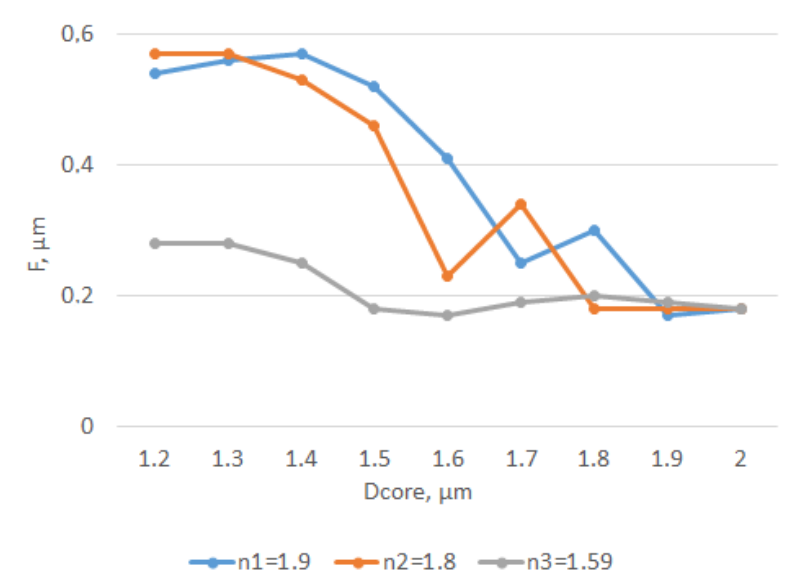

(d)

Fig.4 Dependence of the $I_{\max }(a)$, DOF (b), FWHM (c), F (d) on the core diameter for TM-wave.

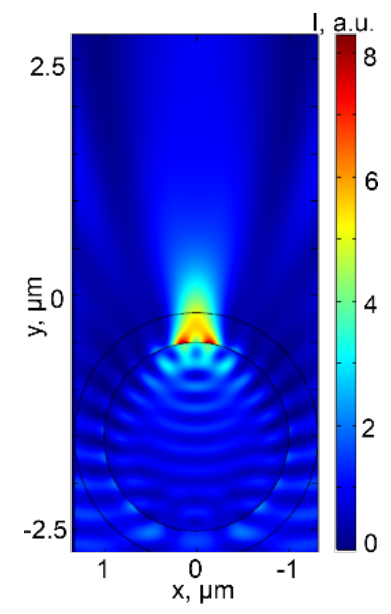

(a)

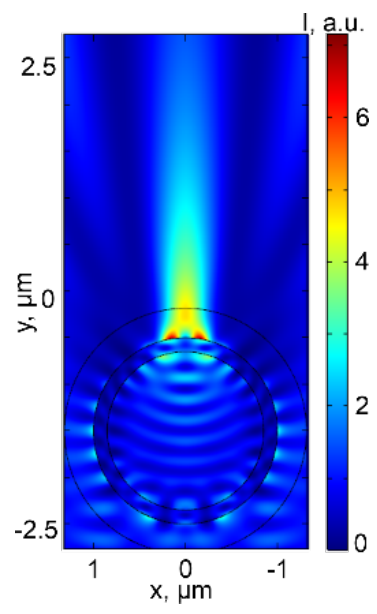

(b)

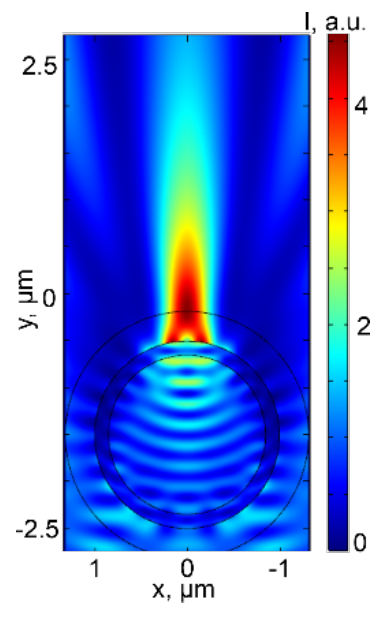

(c)

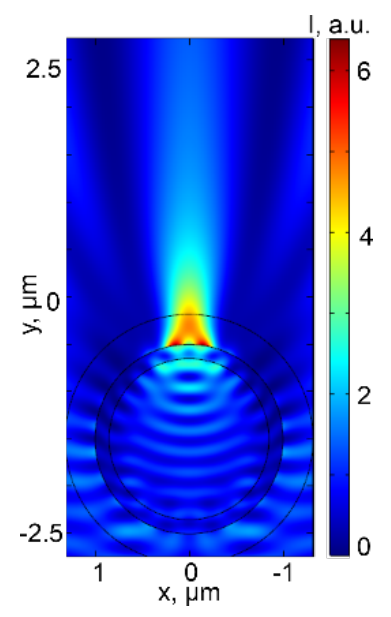

(d)

Fig. 5 The intensity distribution for ordinary microcylinder (a) and two-layered micrcylinders with $\mathrm{n}_{2}=1.9$ and $\mathrm{D}_{\text {core }}=1.7(\mathrm{~b}) ; \mathrm{n}_{2}=1.8$ and $\mathrm{D}_{\text {core }}=1.7(\mathrm{c}) ; \mathrm{n}_{2}=1.59$ and $\mathrm{D}_{\text {core }}=1.7$ (d) illuminated by TM-wave.

It can be seen from Fig. 4 that the influence of shell on focusing process is not so significant as in the case of TE-polarization. Shells with high refractive indeces lead to decreasing of the maximum intensity. Fig. 4(b) shows that thick coatings of high refractive index lead to decreasing of the DOF and the optimal value of shell thickness is about $200-400 \mathrm{~nm}$. It can be seen from Fig. 4(d) that all shells lead to increasing of the focal length. It should be mentioned that the polystyrene shell 
does not significantly affects on all parameters of the nanojet.

Table 4 Parameters of the focal spot formed by the twolayered microcylinders with $\mathrm{n}_{1}=1.45$ and $\mathrm{n}_{2}=1.9$.

\begin{tabular}{ccccc}
$\mathbf{D}_{\text {core, }} \boldsymbol{\mu m}$ & $\mathbf{I}_{\mathbf{m a x}}, \mathbf{a . u}$. & DOF, $\boldsymbol{\lambda}$ & $\mathbf{F W H M , ~} \boldsymbol{\lambda}$ & $\mathbf{F}, \boldsymbol{\mu m}$ \\
\hline 2.0 & 5.56 & 0.82 & 0.70 & 0.18 \\
\hline 1.9 & 4.62 & 1.12 & 0.68 & 0.17 \\
\hline 1.8 & 4.86 & 1.11 & 0.70 & 0.30 \\
\hline $\mathbf{1 . 7}$ & $\mathbf{4 . 7 0}$ & $\mathbf{2 . 4 3}$ & $\mathbf{0 . 6 8}$ & $\mathbf{0 . 2 5}$ \\
\hline 1.6 & 4.41 & 2.22 & 0.70 & 0.41 \\
\hline 1.5 & 4.09 & 1.98 & 0.70 & 0.52 \\
\hline 1.4 & 4.36 & 1.96 & 0.70 & 0.57 \\
\hline 1.3 & 4.34 & 1.75 & 0.71 & 0.56 \\
\hline 1.2 & 1.83 & 1.60 & 0.71 & 0.54
\end{tabular}

Table 5 Parameters of the focal spot formed by the twolayered microcylinders with $\mathrm{n}_{1}=1.45$ and $\mathrm{n}_{2}=1.8$.

\begin{tabular}{ccccc}
$\mathbf{D}_{\text {core, }} \boldsymbol{\mu m}$ & $\mathbf{I}_{\text {max }}, \mathbf{a . u}$ & $\mathbf{D O F}, \boldsymbol{\lambda}$ & $\mathbf{F W H M}, \boldsymbol{\lambda}$ & $\mathbf{F}, \boldsymbol{\mu m}$ \\
\hline 2.0 & 5.56 & 0.82 & 0.70 & 0.18 \\
\hline 1.9 & 4.82 & 1.07 & 0.68 & 0.18 \\
\hline 1.8 & 5.00 & 0.84 & 0.74 & 0.18 \\
\hline $\mathbf{1 . 7}$ & $\mathbf{4 . 5 4}$ & $\mathbf{2 . 1 6}$ & $\mathbf{0 . 6 8}$ & $\mathbf{0 . 3 4}$ \\
\hline 1.6 & 5.08 & 2.36 & 0.71 & 0.23 \\
\hline 1.5 & 4.19 & 2.44 & 0.73 & 0.46 \\
\hline 1.4 & 4.36 & 2.31 & 0.70 & 0.53 \\
\hline 1.3 & 4.60 & 2.15 & 0.71 & 0.57 \\
\hline 1.2 & 4.09 & 1.94 & 0.74 & 0.57
\end{tabular}

It can be seen from the Table 4-6 and Fig. 4 that the two-layered microcylinders form a nanojet with a maximum DOF of $1.44 \lambda$, which is almost 3 times greater than the maximum intensity of the nanojet formed by a conventional dielectric cylinder. At the same time the FWHM was preserved and a decrease in the maximum intensity of the focal spot is about $20 \%$ of the maximum value. The distribution of intensity obtained while focusing of the TMpolarized light by the ordinary cylinder with $\mathrm{n}_{1}=1.45$ and by two-layered cylinders with optimal ratio of the core and the shell is presented in Fig. 5.

Table 6 Parameters of the focal spot formed by the twolayered microcylinders with $\mathrm{n}_{1}=1.45$ and $\mathrm{n}_{2}=1.59$.

\begin{tabular}{ccccc}
$\mathbf{D}_{\text {core, }} \boldsymbol{\mu \mathbf { m }}$ & $\mathbf{I}_{\mathbf{m a x}}, \mathbf{a . u}$. & $\mathbf{D O F}, \boldsymbol{\lambda}$ & $\mathbf{F W H M}, \boldsymbol{\lambda}$ & $\mathbf{F}, \boldsymbol{\mu m}$ \\
\hline 2.0 & 5.56 & 0.82 & 0.70 & 0.18 \\
\hline 1.9 & 5.26 & 0.94 & 0.71 & 0.19 \\
\hline 1.8 & 4.86 & 1.11 & 0.71 & 0.20 \\
\hline $\mathbf{1 . 7}$ & $\mathbf{4 . 7 6}$ & $\mathbf{1 . 2 7}$ & $\mathbf{0 . 6 8}$ & $\mathbf{0 . 1 9}$ \\
\hline 1.6 & 5.03 & 1.18 & 0.71 & 0.17 \\
\hline 1.5 & 5.41 & 0.90 & 0.71 & 0.18 \\
\hline 1.4 & 5.46 & 0.96 & 0.71 & 0.25 \\
\hline 1.3 & 5.30 & 1.19 & 0.69 & 0.28 \\
\hline 1.2 & 5.24 & 1.31 & 0.74 & 0.28
\end{tabular}

It can be seen from Fig. 5 that using a shell makes it possible to form a single peak, in contrast to using a uniform microcylinder with a refractive index of 1.45, which forms a focal spot with two aligned peaks. In addition, nanojets obtainedin the case of using a twolayered microcylinder has a more elongated shape. Moreover, they are located at a considerable distance from the boundary of the cylinder.

\subsection{Comparison of numerical results with FDTD-method}

In this section, the results of numerical modeling for cylinders with a similar design are shown. But in this case FDTD-method implemented in the FullWAVE software package was used for numerical calculations. The results of light focusing by microcylinders with the best designs are presented in Table 7.

Table 7 Parameters of the focal spot formed by ordinary microcylinders and two-layered microcylinders with the best designs.

\begin{tabular}{|c|c|c|c|c|c|c|c|}
\hline Polarization & $\mathrm{n}_{1}$ & $\mathbf{n}_{2}$ & $\mathbf{D}_{\text {core }}, \boldsymbol{\mu m}$ & $I_{\max }$, a.u. & DOF, $\lambda$ & FWHM, $\lambda$ & $\mathrm{F}, \boldsymbol{\mu m}$ \\
\hline \multirow{4}{*}{$\mathrm{TE}$} & 1.45 & - & 2.0 & 8.18 & 0.73 & 0.32 & 0.00 \\
\hline & 1.45 & 1.9 & 1.6 & 5.03 & 1.13 & 0.33 & 0.60 \\
\hline & 1.45 & 1.8 & 1.5 & 4.17 & 2.02 & 0.51 & 0.39 \\
\hline & 1.45 & 1.59 & 1.9 & 11.12 & 0.51 & 0.25 & 0.00 \\
\hline \multirow{4}{*}{$\mathrm{TM}$} & 1.45 & - & 2.0 & 5.53 & 0.85 & 0.70 & 0.15 \\
\hline & 1.45 & 1.9 & 1.7 & 5.05 & 1.58 & 0.70 & 0.31 \\
\hline & 1.45 & 1.8 & 1.7 & 4.89 & 0.74 & 0.44 & 0.48 \\
\hline & 1.45 & 1.59 & 1.7 & 4.63 & 1.36 & 0.45 & 0.64 \\
\hline
\end{tabular}


Table 7 shows that the results obtained using FullWAVE based on FDTD method are in good agreement with the results of COMSOL Multiphysics based on FEM.

The obtained results have a big potential in different biomedicine applications [48-50]. In particular, one of the most important stages of sample preparation in biomedical research is cell separation [48]. It is necessary to purify the target cell population and minimize intercellular interactions prior to biomedical analysis. An optical tweezer or an optical scalpel which is laser radiation focused into a nanojet can be used to separate cells [51]. The fluorescence-based optical detection and imaging technologies are widely used in biomedicine [49]. However, low fluorescence intensity decreases the detection sensitivity and the image brightness. Photonic nanojets can concentrate an incident light in the subwavelength rangeforming a narrow highenergy beam and, thus, increase energy density and enhance fluorescence [52]. The extensive results on the nanofabrication of plasmonic structures stimulated the usage of photonic nanojets for patterning substrates suitable for surface-enhanced spectroscopy, specifically, surface-enhanced Raman scattering (SERS) and surfaceenhanced infrared absorption (SEIRA) [50].

\section{Conclusions}

In this paper, the process of laser light focusing by twolayer dielectric microcylinders of the circular cross-section is investigated. For numerical modeling COMSOL Multiphysics based on the finite element method (FEM) was chosen. Obtained results were verified by FullWAVE based on the finite difference time domain method (FDTD method). We investigated the effect of cladding on the focusing of TE- and TM-polarized laser light with a wavelength of $633 \mathrm{~nm}$. The refractive index of the core was fixed at 1.45 (fused silica glass [45]), while the different refractive indices for the cladding were taken into account: 1.9 (SiN [46])), 1.8 (titanium oxide-organic polymer [47]), and 1.59 (polystyrene [45]). It has been shown that claddings with a higher refractive index than the core refractive index make it possible to increase the depth of the focus and shift the focus away from the cylinder boundary. For example, the design with a shell refractive index of $\mathrm{n}_{2}=1.8$ can increase the depth of the field 3 times for both TE and TM waves. At the same time, if the difference between the refractive indices of the core and the cladding is not too large a two-layer microcylinder can focus the TE-wave in a narrow focal spot the spatial parameters of which are extremely small. For example, a two-layer microcylinder with the core and the shell refractive indices of 1.45 and 1.59 , respectively, formed a more compact focal spot $(\mathrm{FWHM}=0.32 \lambda, \mathrm{DOF}=0.16 \lambda)$ with an intensity $\left(\mathrm{I}_{\max }=11.12\right.$ a.u.) 1.4 times higher than the intensity of the focus formed by an ordinary cylinder with a refractive index $n=1.45$. The proposed designs of two-layer microcylinders are easier to manufacture than designs presented in Refs. [31, 53].

The obtained results can find application in various fields such as nanosensorics [54], nanolithography [55], and microscopy [56], and biomedicine [48-50]. As it was mentioned before, the investigation of the photonic nanojet formation can be useful for successful in all stages of biofluids and biosamples [57] study:from preparation [51] to imaging [52].

\section{Disclosures}

All authors declare that there is no conflict of interests in this paper.

\section{Acknowledgments}

This work was supported by the Russian Foundation for Basic Research RFBR (Grant No. 18-29-20003) in the part of TE-polarization, the Russian Science Foundation (Grant No. 18-19-00595) in the part of TM-polarization, and the Ministry of Science and Higher Education in the framework of works on the State task of the Federal Research Center "Crystallography and Photonics" RAS in the parts "Introduction" and "Conclusion".

\section{References}

1. S. Zhou, "Effects of light polarization in photonic nanojet," Optical and Quantum Electronics 51, 112 (2019).

2. V. V. Kotlyar, S. S. Stafeev, and A. A. Kovalev, "Sharp focusing of a light field with polarization and phase singularities of an arbitrary order," Computer Optics 43(3), 337-346 (2019).

3. A. Darafsheh, "Photonic nanojets and their applications," Journal of Physics: Photonics 3(2), 022001 (2021).

4. J. Zhu, L. L. Goddard, "All-dielectric concentration of electromagnetic fields at the nanoscale: the role of photonic nanojets," Nanoscale Advances 1, 4615-4643 (2019).

5. I. V. Minin, C.-Y. Liu, Y. E. Geints, and O. V. Minin, "Recent Advances in Integrated Photonic Jet-Based Photonics," Photonics 7(2), 41 (2020).

6. X. Chen, M. E. Kandel, and G. Popescu, "Spatial light interference microscopy: principle and applications to biomedicine," Advances in Optics and Photonics 13(2), 353-425 (2021).

7. S. Gao, K. Meng, Z. Yang, H. Liu, F. Wang, L. Sun, and T. Chen, "The Probe-combined Microspheres Applied in Biomedical Field for Super-resolution Imagings and Micromanipulations," In 4th Asia-Pacific Conference on Intelligent Robot Systems (ACIRS), 155-158 (2019).

8. A. Shakhov, A. Astafiev, and V. Nadtochenko, "Microparticle manipulation using femtosecond photonic nanojetassisted laser cavitation," Optics Letters 43(8), 1858 (2018). 
9. S. Surdo, M. Duocastella, and A. Diaspro, "Nanopatterning with Photonic Nanojets: Review and Perspectives in Biomedical Research," Micromachines 12(3), 256 (2021).

10. D. Lu, M. Pedroni, L. Labrador-Paez, M. I. Marques, D. Jaque, and P. Haro-Gonzalez, "Nanojet Trapping of a Single Sub-10 nm Upconverting Nanoparticle in the Full Liquid Water Temperature Range," Nano Micro Small 17(7), 2006764 (2021).

11. R. Ali, F. A. Pinheiro, R. Dutra, F. Rosa, and P. A. M. Neto, "Enantioselective manipulation of single chiral nanoparticles using optical tweezers," Nanoscale 12(8), 5031-5037 (2020).

12. X. Shan, F. Wang, D. Wang, S. Wen, C. Chen, X. Di, P. Nie, J. Liao, Y. Liu, L. Ding, P. J. Reece, and D. Jin, “Optical tweezers beyond refractive index mismatch using highly doped upconversion nanoparticles," Nature Nanotechnology 16, 531-537 (2021).

13. J. L. Killian, F. Ye, and M. D. Wang, “Optical Tweezers: A Force to Be Reckoned With,” Cell 175(6), 1445-1448 (2018).

14. W. Zhang, H. Lei, "Fluorescence enhancement based on cooperative effects of a photonic nanojet and plasmon resonance," Nanoscale 12(12), 6596-6602 (2020).

15. O. V. Polschikova, A. S. Machikhin, A. G. Ramazanova, I. A. Bratchenko, V. E. Pozhar, I. V. Danilycheva, O. R. Katunina, and M. V. Danilychev, "An Acousto-Optic Hyperspectral Unit for Histological Study of Microscopic Objects," Optics and Spectroscopy 125(6), 1074-1080 (2018).

16. L. A. Bratchenko, I. A. Bratchenko, Y A. Khristoforova, D. N. Artemyev, D. Y. Konovalova, P. A. Lebedev, and V. P. Zakharov, "Raman spectroscopy of human skin for kidney failure detection," Journal of Biophotonics 14(6), e202000360 (2020).

17. Z. Zhen, Y. Huang, Y. Feng, Y. Shen, and Z. Li, “An ultranarrow photonic nanojet formed by an engineered twolayer microcylinder of high refractive-index materials," Optics Express 27(6), 9178-9188 (2019).

18. A. Darafsheh, D. Bollinger "Systematic study of the characteristics of the photonic nanojets formed by dielectric microcylinders," Optics Communications 402, 270-275 (2017).

19. A. Darafsheh, D. Bollinger, "Photonic nanojet properties of dielectric microcylinders," Proceedings of SPIE 10106 , 101061U (2017).

20. Y. E. Geints, A. A. Zemlyanov, I. V. Minin, and O. V. Minin, "Overcoming refractive index limit of mesoscale light focusing by means of specular-reflection photonic nanojet," Optics Letters 45(14), 3885-3888 (2020).

21. S. Zhou, T. Zhou, "An ultra-narrow photonic nanojet generated from a high refractive-index micro-flatended cylinder," Applied Physics Express 13, 042010 (2020).

22. A. A. Savelyeva, E. S. Kozlova, "Simulation of laser light focusing with two-layer dielectric microcylinders," Computer Optics 45(2), 208-213 (2021).

23. V. D. Zaitsev, S. S. Stafeev, "The photonic nanojets formation by two-dimensional microprisms," Computer Optics 44(6), 909-916 (2020).

24. A. Abramov, Y. Yue, M. Wang, Z. Wang, and Y. Xu, "Numerical Modeling of Photonic Jet behind Triangular Prism," Asian Journal of Research and Reviews in Physics 4(1), AJR2P.63969 (2021).

25. S. Zhou, K. Li, and Y. Wang, "Tunable photonic nanojets from a micro-cylinder with a dielectric nano-layer," Optik 225,165878 (2021).

26. Y. Cao, Z. Liu, and O. V. Minin, "Deep Subwavelength-Scale Light Focusing and Confinement in NanoholeStructured Mesoscale Dielectric Spheres," Nanomaterials 9(2), 186 (2019).

27. D. Grojo, N. Sandeau, L. Boarino, C. Constantinescu, N. De Leo, M. Laus, and K. Sparnacci, "Bessel-like photonic nanojets from core-shell sub-wavelength spheres," Optics Letters 39(13), 3989 (2014).

28. A. A. Savelyeva, E. S. Kozlova, "Simulation of laser light focusing by a dielectric nanocylinder with gold core," Journal of Physics: Conference Series 1368, 022030 (2019).

29. C.-Y. Liu, K.-L. Hsiao, “Direct imaging of optimal photonic nanojets from core-shell microcylinders," Optics Letters 40(22), 5303-5306 (2015).

30. A. A. Savelyeva, E. S. Kozlova, "Simulation of light focusing by a dielectric microcylinder with a metal film and gap on shadow side," Journal of Physics: Conference Series 1745, 012010, (2021).

31. Y. Huang, Z. Zhen, Y. Shen, C. Min, and G. Veronis, "Optimization of photonic nanojets generated by multilayer microcylinders with a genetic algorithm," Optics express 27(2), 1310-1325 (2019).

32. A. A. Savelyeva, E. S. Kozlova, "Simulation of laser light focusing by two-layered dielectric cylinders," In $22^{\text {nd }}$ International Conference on Transparent Optical Networks, 19-23 July 2020, 1-4 (2020).

33. M. N. O. Sadiku, “A simple introduction to finite element analysis of electromagnetic problems," IEEE Transactions on Education 32(2), 85-93 (1989).

34. D. W. Pepper, J. C. Heinrich, The Finite Element Method: Basic Concepts and Aplications with MATLAB, MAPLE, and COMSOL, $3^{\text {rd }}$ ed., CRC Press, Boca Raton, USA (2017).

35. S. Singh, R. S. Kaler, "Performance analysis of evanescent wave absorption plasmonic optical sensor with COMSOL FEM method simulation,” Procedia Computer Science 125, 376-381 (2018). 
36. V. D. Zaitsev, S. S. Stafeev, "Photonic Jets Arrays Produced by Triangular Dielectric Prisms for Mid-IR Imaging," In 2019 PhotonIcs \& Electromagnetics Research Symposium - Spring (PIERS-Spring), 17-20 June 2019, 26102614 (2019).

37. E. S. Kozlova, V. V. Kotlyar, and S. A. Degtyarev, "Simulation of resonance focusing of a picosecond pulse by a dielectric microcylinder," Computer Optics 39(1), 45-51 (2015).

38. M. A. Born, E. Wolf, Principles of optics Electromagnetic Theory of Propagation, Interference, and Diffraction of Light, Cambridge University Press, London (1999).

39. K. S. Yee, "Numerical Solution of Initial Boundary Value Problems Involving Maxwell's Equations in Isotropic Media," In IEEE Transactions on Antennas and Propagation 14(3), 302-307 (1966).

40. S. D. Gedney, "Introduction to the Finite-Difference Time-Domain (FDTD) Method for Electromagnetics," Synthesis Lectures on Computational Electromagnetics 6(1), 1-250 (2011).

41. X. Guo, Y. Ding, X. Chen, Y. Duan, and X. Ni, "Molding free-space light with guided wave-driven metasurfaces," Science Advances 6(29), eabb4142 (2021).

42. A. G. Nalimov, "Energy flux of a vortex field focused using a secant gradient lens," Computer Optics 44(5), 707$711(2020)$.

43. E. S. Kozlova, S. S. Stafeev, S. A. Fomchenkov, V. V. Podlipnov, and V. V. Kotlyar, "Laser Light Focusing by Aluminium Zone Plate," In 22 ${ }^{\text {nd }}$ International Conference on Transparent Optical Networks, 1-4 (2020).

44. S. Piltyay, A. Bulashenko, Y. Herhil, and O. Bulashenko, "FDTD and FEM Simulation of Microwave Waveguide Polarizers," In IEEE $2^{\text {nd }}$ International Conference on Advanced Trends in Information Theory, 357-363 (2020).

45. J. F. Shackelford, Introduction to Materials Science for Engineers, $5^{\text {th }}$ ed., McGraw-Hill, New York (2000).

46. H. Nagel, A. G. Aberle, and R. Hezel, "Optimised antireflection coatings for planar silicon solar cells using remote PECVD silicon nitride and porous silicon dioxide," Progress in Photovoltaics: Research and Applications 7(4), 245260 (1999).

47. T. D. Flaim, Y. Wang, and R. Mercado, "High-refractive-index polymer coatings for optoelectronics applications," Advances in Optical Thin Films 5250, 423-434 (2004).

48. N. Liu, C. Petchakup, H. M. Tay, K. H. H. Li, and H. W. Hou, "Spiral Inertial Microfluidics for Cell Separation and Biomedical Applications," Cell Culture Techniques, 99-150 (2019).

49. W. Zhang, H. Lei, "Fluorescence enhancement based on cooperative effects of a photonic nanojet and plasmon resonance," Nanoscale 12(12), 6596-6602 (2020).

50. S. Surdo, M. Duocastella and A. Diaspro, "Nanopatterning with Photonic Nanojets: Review and Perspectives in Biomedical Research," Micromachines 12(3), 256 (2021).

51. A. A. R. Neves, "Photonic nanojets in optical tweezers. Journal of Quantitative Spectroscopy and Radiative Transfer," Journal of Quantitative Spectroscopy and Radiative Transfer 162, 122-132 (2015).

52. W. Liu, X. Li, Y. Song, C. Zhang, X. Han, H. Long, B. Wang, K. Wang, and P. Lu, “Cooperative Enhancement of Two-Photon-Absorption-Induced Photoluminescence from a 2D Perovskite-Microsphere Hybrid Dielectric Structure," Advanced Functional Materials 28(26), 1707550 (2018).

53. H. Zhang, "Enhanced subwavelength photonic nanojet focusing via a graded-index round-head microcylinder," Optik 203, 163973 (2020).

54. S. Cheng, X. Zhang, W. Ma, and S. Tao, "Detecting a Zeptogram of Pyridine with a Hybrid Plasmonic-Photonic Nanosensor," ACS Sens 4(3), 586-594 (2019).

55. J. Wei, K. Zhang, T. Wei, Y. Wang, Y. Wu, and M. Xiao, "High-speed maskless nanolithography with visible light based on photothermal localization,” Scientific Reports 7, 43892 (2017).

56. R. Tenne, U. Rossman, B. Rephael, Y. Israel, A. Krupinski-Ptaszek, R. Lapkiewicz, Y. Silberberg, and D. Oron, "Super-resolution enhancement by quantum image scanning microscopy," Nature Photonics 13, 116-122 (2019).

57. L. A. Bratchenko, I. A. Bratchenko, A. A. Lykina, M. V. Komarova, D. N. Artemyev, O. O. Myakinin, A. A. Moryatov, I. L. Davydkin, S. V. Kozlov, and V. P. Zakharov, "Comparative study of multivariative analysis methods of blood Raman spectra classification,” Journal of Raman Spectroscopy 51(2), 279-292 (2019). 